\title{
On the experimental intradiscal pressure measurement techniques: a review
}

\author{
A. Araújo ${ }^{1}$, N. Peixinho ${ }^{2}$, A. Pinho ${ }^{3}$, J.C.P. Claro ${ }^{4}$ \\ ${ }^{1}$ University of Minho, Portugal, e-mail: angeloaraujo@dem.uminho.pt \\ ${ }^{2}$ University of Minho, Portugal, e-mail: peixinho@dem.uminho.pt \\ ${ }^{3}$ University of Minho, Portugal, e-mail: acmpinho@dem.uminho.pt \\ ${ }^{4}$ University of Minho, Portugal, e-mail: jcpclaro@dem.uminho.pt
}

\begin{abstract}
The intradiscal pressure has been essential for prevent the spinal complaints by forming a basis for clinical advice to promote the correct sitting postures. As a consequence, it is evident the need of an accurate method for measure the intradiscal pressure, to better understand the disc response to hydorstatic pressure fluctuations. Numerous reviews regarding disc mechanics are available, including intradiscal pressure benchmarks; however, an analysis on the techniques of intradiscal pressure measurement is needed. Therefore, this review will remain focused on the methodologies adopted for measure the intradiscal pressure in several conditions: for different daily activities, under external loads and for values where occurs annulus fibrosus disruption. The importance of the intradiscal pressure on disc function will be discussed as well as the some guidelines for design new measurement techniques will be defined.
\end{abstract}

Key words: Intervertebral Disc, Intradiscal Pressure.

\section{Introduction}

The intervertebral disc (IVD) is a fibrocartilage structure located between two vertebral bodies, which is surrounded by ligaments and muscles ${ }^{23}$. This intricate organization is comprised by a peripheral angle-ply laminate ring, the annulus fibrosus (AF) with a gelatinous nucleus in its center (NP), and it is limited above and beyond by the cartilaginous endplates (CEP) $)^{14}$ (Fig. 1).

The IVD plays an important role at spinal level: it is responsible for the spine motion, helping the spine on the weight support and load transfer from head and upper torso to the pelvis ${ }^{15,28}$. The IVD is specially designed to perform these functions, since the mechanical response of disc to loading is time-dependent ${ }^{25}$ : while the short time response is governed by viscoelastic phenomena ${ }^{2,6}$, the long term response is guided by poroelastic and osmotic events ${ }^{20,25}$. 
Moreover, in opposition to the AF generally considered as a fibrous solid ${ }^{1}$, the NP presents an high water content, revealing a fluid-like behavior ${ }^{1}$. Thus, a healthy NP is capable of sustain stress gradients due to hydrostatic pressure exhibited by NP, normally known as intradiscal pressure (IDP) ${ }^{3}$ (Fig. 1).

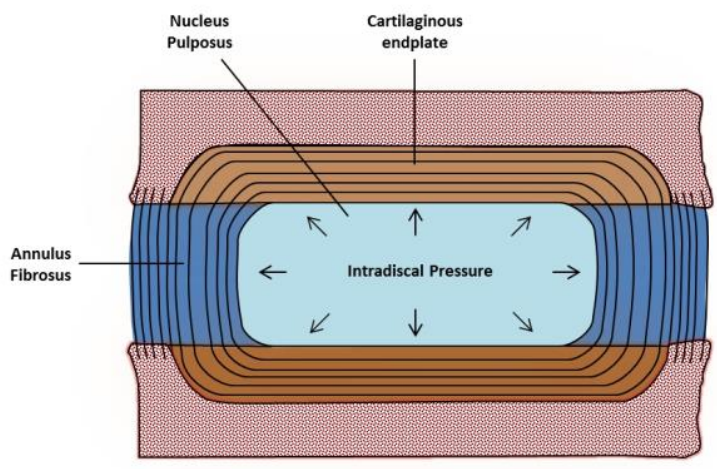

Fig. 1 - Representation of the intervertebral disc and the schematic concept of intradiscal pressure

The IDP varied with body posture and with direct compressive force applied ${ }^{19}$. In this review, the importance of the IDP on intervertebral disc function will be discussed and the criteria for design new IDP measurement techniques will be defined. Subsequent sections describe the previous methodologies developed to determine the IDP in physiological and in failure cases. Finally, it will conclude with some main points the development of new IDP measurement techniques.

\section{The IDP importance for disc function evaluation and properties determination}

The internal disc pressure or intradiscal pressure (IDP) can be defined as the hydrostatic pressure presented by the NP of an healthy IVD ${ }^{3}$. The IDP plays a key role on the IVD's ability to withstand the physiological loads ${ }^{24}$, being an important parameter to understand the spinal on the disc degeneration.

The IDP data has been essential for prevent the spinal complaints by forming a basis for clinical advice to promote the correct sitting postures ${ }^{3}$. The measurements of IDP helps to clarify the effect of the external loads on the IVD behavior ${ }^{3}$ and to recognize the mechanism of IDP drop in disc degeneration. In addition, these data is the basis for physiotherapy and rehabilitation programs ${ }^{29}$.

At a biomechanical point of view, the IDP is highly influenced by the axial spinal load ${ }^{18}$. According to this, an increase on the compressive load applied to healthy discs is converted into IDP ${ }^{19}$. Since the NP can be considered as incom- 
pressible, the AF bulges outward due to the stretch of annular ${ }^{26}$, which, together with osmotic phenomenon, promotes a loss on both disc height and volume.

The importance of IDP is reinforced due to difficult on the assessment of the disc strengthen properties. As example, a simple compressive overloading does not induce damage on the disc structure. Previous studies showed that before occurring any disc disruption, the compressive overloading promotes the vertebral endplate damage and collapse ${ }^{19}$. These phenomena could be explained at a cellular level by the influence of the IDP on the chondroid tissues, characteristic of the IVD ${ }^{1}$. On the one hand, the IDP or gradient pressures could induce the internal disruption on these tissues, causing a progressive structural failure typical on disc degeneration ${ }^{1}$. On the other hand, the stresses and pressures oscillation also affects the cell metabolism, where the IDP levels influences the matrix synthesis ${ }^{1}$. In sum, it is evident the need of an efficient method for measure the IDP, in order to better understand the mechanical behavior of IVD.

\section{On the IDP measurement: a methodology review}

The measurement of the IDP is a subject of intense research. Numerous authors have developed experimental methods to determine the relationship between the IDP and the disc mechanics: some authors were focused on the association between the IDP and the external load applied or posture adopted; others were more centered on the calculation of IDP value that leads to AF disruption. Next subsections will describe with more detail the studies performed under these subjects.

\subsection{Relationship between IDP and posture or exter- nal loading}

Previous studies have investigated the relationship between an external loads applied or posture and IDP, on lumbar ${ }^{5,9,11,12,18,21,29}$ or on cervical ${ }^{4,8,17}$ discs.

The methodologies developed for measure the IDP were diverse (Table 1).

Table 1. Previous studies reporting the IDP according to the type of load/posture adopted

\begin{tabular}{|c|c|c|c|c|}
\hline Author & Year & Transducer Type & IDP evaluation & Mean IDP $(M P a)$ \\
\hline \multicolumn{5}{|c|}{ Lumbar spine } \\
\hline \multirow[t]{2}{*}{ Nachemson } & $1964 / 1965$ & Liquid-filled & Relaxed standing & 0.70 \\
\hline & & & Relaxed sitting & 1.13 \\
\hline \multirow{2}{*}{$\begin{array}{l}\text { Nachemson } \\
\& \text { Elfstrom }\end{array}$} & 1970 & Piezoresistive & Relaxed standing & 0.72 \\
\hline & & & Relaxed sitting & 1.00 \\
\hline Schultz & 1982 & Piezoresistive & $2400 \mathrm{~N}$ axial load & 1.60 \\
\hline
\end{tabular}




\begin{tabular}{|c|c|c|c|c|}
\hline \multirow[t]{2}{*}{ Sato } & 1999 & Piezoresistive & Relaxed standing & 0.53 \\
\hline & & & Relaxed sitting & 0.63 \\
\hline \multirow[t]{3}{*}{ Wilke } & 1999 & Piezoresistive & Relaxed standing & 0.50 \\
\hline & & & Relaxed sitting & 0.46 \\
\hline & & & $\begin{array}{l}\text { Lifting a } 20-\mathrm{kg} \text { weight } \\
\text { with round flexed back }\end{array}$ & 2.30 \\
\hline Heuer & 2007 & Laser scanning & $500 \mathrm{~N}$ axial load & 0.49 \\
\hline Dennison & 2008 & Fibre Gratings & $800 \mathrm{~N}$ axial load ${ }^{1}$ & 2.40 to 3.50 \\
\hline \multicolumn{5}{|c|}{ Cervical spine } \\
\hline \multirow[t]{4}{*}{ Hattori } & 1981 & Piezoresistive & $53 \mathrm{~N}$ axial load & 0.31 \\
\hline & & & $75 \mathrm{~N}$ axial load & 0.45 \\
\hline & & & $100 \mathrm{~N}$ axial load & 0.59 \\
\hline & & & $155 \mathrm{~N}$ axial load & 0.91 \\
\hline \multirow[t]{4}{*}{ Pospiech } & 1999 & Piezoresistive & Muscular inactivation & \\
\hline & & & - Flexion/Extension & $0.23-0.32$ \\
\hline & & & Muscular activation & \\
\hline & & & - Flexion/Extension & $0.36-0.64$ \\
\hline Cripton & 2001 & Piezoresistive & $\sim 1000 \mathrm{~N}$ axial load & 3.5 \\
\hline
\end{tabular}

The first approaches were performed on the $1960 \mathrm{~s}$ and $1970 \mathrm{~s}^{12,13,16}$ on in vivo situation, alerting for the importance of IDP on the spinal biomechanics ${ }^{3}$. A pressure transducer using elastic polyethylene tubing threaded over the side near of a tip from hollow liquid-filled needle, connected with an electromanometer, was used in healthy discs for IDP determination. The data showed that a healthy NP could behave hydrostatically and the IDP is dependent on the posture. Even though the interesting findings, this pioneer approach presents a couple of limitations. First, the polyethylene membrane does not present enough sensitivity for dynamic pressure measurements. Second, the fluid-filled needle is not prepared to bend more than $20^{\circ}$.

The evolution of the transducer technology and its increased accuracy leads to a decrease on the IDP measurement (a reduction of 25 and 33\%) $)^{3}$, due to the replacement of liquid-filled sensors by the piezoresistive ones, as well as the set of calibration to body temperature rather than room temperature ${ }^{3}$.

The initial approach using was developed by Nachemson \& Elfstrom ${ }^{11}$, using a piezoresistive semiconductor strain gauge embebed in a rigid resin into a tip of a $0.8 \mathrm{~mm}$ diameter transducer needle ${ }^{3}$. This sensor allows bending until $40^{\circ}$ without affecting the IDP measurement ${ }^{3}$, increasing the IDP measurement accuracy.

In early $80^{\prime}$ s, Schultz et al. ${ }^{22}$ tried to validate a biomechanical model lumbar spine by monitoring IDP and myoelectric signals, using a piezoresistive transducer for IDP measurement. This study pointed to $1.6 \mathrm{MPa}$ of mean IDP, for a compres- 
sive load as much as $2.4 \mathrm{kN}$. The main finding of this study was that overloading could be a promoter of low back disorders.

In end of the 90s, two important studies were published: Sato et al. ${ }^{18}$ developed a new approach using a piezoresistive sensor to determine the IDP in vivo young patients $(25 \pm 2$ y.o. $)$. The innovative principle of this method is the sensor positioning: the sensing diaphragm was mounted laterally on the transducer needle (1.2-mm diameter); Wilke et al. ${ }^{29}$, studied the IDP in vivo, in one volunteer performing various daily life activities. To measure the IDP, a piezoelectric pressure transducer, with $1.5 \mathrm{~mm}$ diameter and $7 \mathrm{~mm}$ length, was implanted in the NP of a healthy L4-L5 disc. However, in this case, the IDP was record with a telemetrically, avoiding the problem of having a needle in situ. These studies reported similar IDP for standing position and reinforced the idea that in vivo IDP varied according to the adopted position of the body and the compressive force applied.

Although the accuracy of piezoelectric sensors, they are not able to characterize pressure profiles within disc ${ }^{5}$. With the advances on the sensor technology, new sensors were adapted for IDP measurement. Dennison et al. ${ }^{5}$ used small diameter $(125 \mu \mathrm{m})$ fiber-bragg grating, which consist on an optical fiber with a Bragg grating inscribed into a fiber core. These sensors present a biocompatibility, mechanical compliance and insusceptibility to electromagnetic interference. The results reported a linear response of disc pressure to compressive loads.

The IVD bulging was also adopted as an indirect parameter of the internal stress measurement of the disc. Heuer et al. ${ }^{9}$ quantified the IDP ex vivo using a noncontact laser scanning method, which measure a 3D contour. The test consists on the application of $15 \mathrm{~min}$ of a $500 \mathrm{~N}$ static compression. This work showed that these loads results on an IDP of $0.49 \mathrm{MPa}$ (range: $0.36-0.53 \mathrm{MPa}$ ), decreasing linearly to $0.48 \mathrm{MPa}(0.36-0.52 \mathrm{MPa})$ when discs were constantly compressed.

In contrast to the extensive experimental work on lumbar IDP, the data related to cervical pressure is extremely scarce. The measurements on the cervical are challenging due to small size and anatomy of cervical IVDs.

Hattori et al. ${ }^{8}$ was the first study recording the cervical IDP in vivo. A needlebased pressure transducer was used to measure IDP, during common neck movements. The results found no differences between cervical IVDs, detecting the values of $0.31 \mathrm{MPa}$ and $0.91 \mathrm{MPa}$ for $53 \mathrm{~N}$ and $155 \mathrm{~N}$ of axial load, respectively.

Later, Pospiech et al. ${ }^{17}$ studied the IDP of cervical spine in vivo under simulated muscular forces in intact spines as well as in fused specimens. The IDP was measured using a pressure transducer mounted on a $1.3 \mathrm{~mm}$-diameter needle. The results showed significant increase in IDP when the musculature was activated and a marked increase in IDP in both segments adjacent to fusion IVDs.

Alhough the importance of these findings, the cervical IDP measurement presents more difficult, since they cervical annular fibers could disrupt with the needle-tip insertion, due to its rigidity and its large diameter (over $1 \mathrm{~mm}$ ) ${ }^{4}$. To minimizing the AF disruption and reduce the distortion of the IDP signals or specimen kinematic behavior, Cripton et al. $^{4}$ tested ex vivo the cervical IDP response to external loads, using with a $0.26 \mathrm{~mm}$ diameter flexible electric wires passing through 
AF. They found a maximum of $3.5 \mathrm{MPa}$ for cervical IDP, with $1000 \mathrm{~N}$ load, while in lumbar for the same load, it is common an IDP of $1 \mathrm{MPa}$.

\subsection{The annular failure strength due to inflation method pressurization}

Despite the panoply of mechanical testing found on literature intending to elucidate the contribution of loads to annular tear or disruption, the effect of the IDP fluctuation on the AF injury remains largely unknown. The measurement of the IDP value that leads to AF disruption is a subject of extreme interest, not only to understand the mechanisms of IVD failure but also for IVD replacement designing, since it should withstand the daily routine activities without collapsing.

Some studies determined the maximum value of IDP that annular fibers support before failure ${ }^{10,19,27}$. The table 2 reports the failure IDP in previous studies.

Table 2. Previous studies reporting failure pressure data several IVD models.

\begin{tabular}{llll}
\hline Author & Year & Models & Mean failure pressure (MPa) \\
\hline Schetchman & 2006 & Lumbar bovine & $18 \pm 3$ \\
Veres & 2010 & Lumbar ovine & $14.1 \pm 3.9$ \\
Menkowitz & 2005 & Cervical human spine & 0.28 (min-máx:0.1-1.18) \\
\hline
\end{tabular}

Schechtman et al. (2005) investigated the intrinsic failure strength of the intact bovine caudal disc under a simple mode of inflation, using a hydraulic actuator. They injected a colored hydrogel, under monitored pressure, into the NP. It was found a mean hydrostatic failure pressure of $18 \pm 3 \mathrm{MPa}$. This method allowed understanding the alterations of the intrinsic disc strength associated with prior loading history or degeneration. However, it does not give information about the microstructural behavior of inner annular fibers after the inflation.

Later, Veres et al. ${ }^{27}$ used the same technique performed by Schechtman et al. (2005) to investigate the role of high IDP on annular fibers disruption in ovine lumbar IVDs. This team included the analysis of the AF damage after pressure insertion by a microstructural investigation. The main findings showed that posterior annular region is more susceptible to disruption than the other disc regions, due to its inability to distribute hydrostatic pressures circumferentially.

In terms of cervical spine, Menkowitz et al. ${ }^{10}$ documented a mean intradiscal rupture pressure of $0.28 \mathrm{MPa}$ (range $0.1-1.18 \mathrm{MPa}$ ), using a $25 \mathrm{G}$ needle for the insertion of an contrast dye with IDP monitoring during time. This study showed that in cervical spine the injury could be induced at lower pressures. 


\section{The criteria for a new method for intradiscal measurement}

All these methodologies helps to generate valuable data, useful for a preclinically evaluation of disc injuries. However, some difficulties are noticed on the experimental determination of IDP: the direct measurements of IDP through in vivo studies are normally avoided since the insertion of a transducer into the IVD could damage it ${ }^{29}$. Therefore, the technique for in vivo IDP measurement should be non-invasive, since the IDP must be determined without disc disruption. This is essential for in vivo human tests, since the discs should keep its complete functions after the IDP monitoring. New techniques must be also accurate, as the IDP magnitude is low and highly sensitive to movements or load application.

In terms of annular failure strength, more studies should be performed to fully understand the rupture mechanism. Previous studies did not follow-up how the failure occurs: they only assessed the IVD structure after the rupture. Therefore, it is desirable an approach that assesses the disc behavior during the inflation.

A promising method for both in vivo IDP monitoring and the disc inflation follow-up is the use of imaging resources. With the advance of imaging techniques, the assessment of inner IVD pressure is possible, representing a non-invasive approach that could be better explored. In addition, coupling a inflation method with a microstructural assessment real time could bring a new light on poorly understood mechanism of IVD failure related to higher IDP values.

New techniques must also allowing the measurement of IDP on different types of materials inside the IVD, with potential to replace NP, in order to find a material which could has the similar hydrostatic response as NP.

To conclude, notwithstanding the great efforts performed in past for experimental IDP measurement, new approaches and techniques are needed in order to better understand the IDP influence on disc behavior.

\section{Referencing}

1. Adams MA, Dolan P, Mcnally DS. The internal mechanical functioning of intervertebral discs and articular cartilage, and its relevance to matrix biology. Stress Int J Biol Stress. 28:384-389 (2009).

2. Campana S, Charpail E, de Guise J a., Rillardon L, Skalli W, Mitton D. Relationships between viscoelastic properties of lumbar intervertebral disc and degeneration grade assessed by MRI. $J$ Mech Behav Biomed Mater. (2011)

3. Claus A, Hides J, Moseley GL, Hodges P. Sitting versus standing: does the intradiscal pressure cause disc degeneration or low back pain? J Electromyogr Kinesiol. 18(4):550-8 (2008).

4. Cripton $\mathrm{P}$ a, Dumas $\mathrm{G}$ a, Nolte LP. A minimally disruptive technique for measuring intervertebral disc pressure in vitro: application to the cervical spine. J Biomech. 34(4):545-9 (2001).

5. Dennison CR, Wild PM, Byrnes PWG, et al. Ex vivo measurement of lumbar intervertebral disc pressure using fibre-Bragg gratings. J Biomech. 41(1):221-5 (2008). 
6. Ellingson AM, Nuckley DJ. Intervertebral disc viscoelastic parameters and residual mechanics spatially quantified using a hybrid confined/in situ indentation method. $J$ Biomech. 45(3):491-6 (2012).

7. Hattori S., Oda H, Kawai S U-S. Cervical intradiscal pressure in movements and traction of the cervical spine. Z Orthop. 19:568-569 (1981).

8. Heuer F, Schmitt H, Schmidt H, Claes L, Wilke H-J. Creep associated changes in intervertebral disc bulging obtained with a laser scanning device. Clin Biomech. 22(7):737-744 (2007).

9. Menkowitz M, Stieber JR, Wenokor C, Cohen JD, Donald GD, Cresanti-Dakinis C. Intradiscal pressure monitoring in the cervical spine. Pain Physician. 8(2):163-6 (2005).

10. Nachemson A, Elfström G. Intravital dynamic pressure measurements in lumbar discs. A study of common movements, maneuvers and exercises. Scand J Rehabil Med Suppl. 1970;1:1-40.

11. Nachemson A. The effect of forward leaning on lumbar intradiscal pressure. Acta Orthop Scand. 35:314-28 (1965)

12. Nachemson A. The influence of spinal movements on the lumbar intradiscal pressure and on the tensil stresses in the annulus fibrosus. Acta Orthop Scand. 33:183-207 (1963);

13. Nerurkar NL, Elliott DM, Mauck RL. Mechanical design criteria for intervertebral disc tissue engineering. J Biomech. 43:1017-1030 (2010);

14. Niosi CA, Oxland TR. Degenerative mechanics of the lumbar spine. Spine J.;4(6 Suppl):202S208S (2004)

15. Okushima H. [Study on hydrodynamic pressure of lumbar intervertebral disc]. Nihon Geka Hokan;39(1):45-57(1970).

16. Pospiech J, Stolke D, Wilke HJ, Claes LE. Intradiscal pressure recordings in the cervical spine. Neurosurgery. 44(2):379-385 (1999).

17. Sato K, Kikuchi S, Yonezawa T. In vivo intradiscal pressure measurement in healthy individuals and in patients with ongoing back problems. Spine (Phila Pa 1976). 24(23):2468-74 (1999).

18. Schechtman H, Robertson PA, Broom ND. Failure strength of the bovine caudal disc under internal hydrostatic pressure. J Biomech. 39(8):1401-1409 (2006).

19. Schroeder Y, Wilson W, Huyghe JM, Baaijens FPT. Osmoviscoelastic finite element model of the intervertebral disc. Eur Spine J. Suppl 3:S361-71 (2006).

20.Schultz A, Andersson G, Ortengren R, Haderspeck K, Nachemson A. Loads on the lumbar spine. Validation of a biomechanical analysis by measurements of intradiscal pressures and myoelectric signals. J Bone Joint Surg Am. 64(5):713-20 (1982).

21. Schultz A, Andersson G, Ortengren R, Haderspeck K, Nachemson A. Loads on the lumbar spine. Validation of a biomechanical analysis by measurements of intradiscal pressures and myoelectric signals. J Bone Joint Surg Am. 64(5):713-20 (1982).

22. Silva-Correia J, Correia SI, Oliveira JM, Reis RL. Tissue engineering strategies applied in the regeneration of the human intervertebral disk. Biotechnol Adv. 31(8):1514-31 (2013).

23. Steffen T, Baramki HG, Rubin R, Antoniou J, Aebi M. Lumbar intradiscal pressure measured in the anterior and posterolateral annular regions during asymmetrical loading. Clin Biomech (Bristol, Avon). 13(7):495-505 (1998)

24. Stokes IF, Laible JP, Gardner-Morse MG, Costi JJ, Iatridis JC. Refinement of elastic, poroelastic, and osmotic tissue properties of intervertebral disks to analyze behavior in compression. Ann Biomed Eng. 39(1):122-31 (2011).

25. Van der Veen AJ, Mullender MG, Kingma I, van Dieen JH, Van JH, Smit TH. Contribution of vertebral bodies, endplates, and intervertebral discs to the compression creep of spinal motion segments. J Biomech. 41(6):1260-8 (2008).

26. Veres SP, Robertson PA, Broom ND. The influence of torsion on disc herniation when combined with flexion. Eur spine J. 19(9):1468-1478 (2010).

27. White, Augustus A., Panjabi MM. Clinical Biomechanics of the Spine. Lippincott Williams \& Wilkins; (1990).

28. Wilke HJ, Neef P, Caimi M, Hoogland T, Claes LE. New in vivo measurements of pressures in the intervertebral disc in daily life. Spine (Phila Pa 1976). 24(8):755-762 (1999). 